\title{
A fuzzy logic approach to modelling the passengers' flow and dwelling time
}

\author{
A. Berbey ${ }^{1}$, R. Galan ${ }^{2}$, J. D. Sanz Bobi ${ }^{2}$ \& R. Caballero ${ }^{1}$ \\ ${ }^{1}$ Universidad Tecnológica de Panamá, Panama, Republic of Panama \\ ${ }^{2}$ Universidad Politécnica de Madrid, Madrid, Spain
}

\begin{abstract}
The passengers' flow and station dwell time estimation are important tasks for mass transit planning. However, classical methods are difficult to apply into some practical achievements. This paper presents a new approach that models passengers' flow and its effect on passenger alighting and boarding time in mass transportation systems in the presence of uncertainties. The applied technique combines origin destination matrices approach with the application of artificial intelligence. This new approach allows the inclusion of some intuitive knowledge provided by a fuzzy logic inference motor to predict the flow demand of passengers' trips, alighting and boarding time passenger cars in explicit stations.
\end{abstract}

Keywords: passengers' flow modelling, fuzzy logic inference, artificial intelligence, uncertainties.

\section{Introduction}

It is well known that passengers' flow modelling is very important for evaluating not only the effectiveness of station facilities, but also the rolling stock [1]. This model reflects the influence of these two factors in the process of boarding and alighting passengers on the train. On the other hand, it is important to note that the correct estimation of dwelling time is as important as the restrictions imposed by the line speed and acceleration of the train [2].

The passengers' flow modelling has been tackled by several authors [3-7]. In order to estimate the dwelling time, different models have been proposed. Most of them involve passengers' flow calculation in the process of boarding and alighting within each train car $[1,2,8]$. 
However, it has been shown that these methods have limitations in certain scenarios. Thus, field studies have shown that the passengers' flow is not homogeneous among the different boarding and alighting zones [2, 9]. Furthermore, the distribution of this flow of passengers has proved to be not only a function of access facilities on platforms, but also the dynamics of interaction of different groups of people who share a small space. The modelling of behaviour of these human groups is complex, since it depends heavily on gender, age, profession, among other factors.

There are several approaches to model human behaviour. However, the use of fuzzy logic has proven to be a promising tool because it can easily integrates the railway planning experts' experience and models passengers' behaviour $[4,6,10]$.

\section{Estimating the distribution of passengers on the train by OD matrices}

The matrix method of area trips O-D (Origin-Destination) is one of the most used methods to design the movement of passengers [11]. This method can be used to model passenger travel between different stations. However, due to economic and practical reasons this model is not regarded as passengers are distributed along the various train cars. Consequently, if it is necessary to estimate passengers' distribution on train, it is necessary to generate another level of OD matrix at train car, in every station. This new OD matrix can be constructed by subdividing the original OD matrix,

\begin{tabular}{|c|c|c|c|c|}
\hline$\ddots$ & $\mathrm{T}_{12}$ & $\ldots$ & $\mathrm{T}_{1 \mathrm{n}}$ & $\mathrm{O}_{\mathrm{T} 1}$ \\
\hline $\mathrm{T}_{21}$ & $\ddots$ & $\cdots$ & $\mathrm{T}_{2 \mathrm{n}}$ & $\mathrm{O}_{\mathrm{T} 2}$ \\
\hline$\cdots$ & $\cdots$ & $\ddots$ & $\cdots$ & $\cdots$ \\
\hline $\mathrm{T}_{\mathrm{n} 1}$ & $\mathrm{~T}_{\mathrm{n} 2}$ & $\cdots$ & $\ddots$ & $\mathrm{O}_{\mathrm{Tn}}$ \\
\hline $\mathrm{D}_{\mathrm{T} 1}$ & $\mathrm{D}_{\mathrm{T} 2}$ & $\cdots$ & $\mathrm{D}_{\mathrm{Tn}}$ & \\
\hline
\end{tabular}

Figure 1: $\quad$ Original $T$ OD matrix.

where $D_{T} \in \mathbb{R}^{1 \times n}$ is the alighting vector and $O_{T} \in \mathbb{R}^{n \times 1}$ corresponds to the boarding vector of passenger for $n$ stations. Here, each element of alighting $D_{T J}$ 
and boarding $O_{T I}$ vectors can be subdivided into $m$ passenger cars, becoming two new vectors $D \in \mathbb{R}^{1 \times n_{s}}$ and $O \in \mathbb{R}^{n_{s} \times 1}$ where:

$$
\begin{aligned}
& O=\left[\begin{array}{lllllllllllll}
O_{11} & O_{12} & \ldots & O_{1 m} & O_{21} & O_{22} & \ldots & O_{2 m} & \ldots & O_{n 1} & O_{n 2} & \ldots & O_{n m}
\end{array}\right] \\
& D=\left[\begin{array}{lllllllllllll}
D_{11} & D_{12} & \ldots & D_{1 m} & D_{21} & D_{22} & \ldots & D_{2 m} & \ldots & D_{n 1} & D_{n 2} & \ldots & D_{n m}
\end{array}\right] \\
& n_{s}=n m
\end{aligned}
$$

Also, the trip matrix $T \in \mathbb{R}^{n \times n}$ is subdivided, resulting in a matrix that

\begin{tabular}{|c|c|c|c|c|c|c|c|c|c|c|c|}
\hline \multirow{3}{*}{\multicolumn{3}{|c|}{$\ddots$}} & $\mathrm{M}_{1121}$ & $\ldots$ & $\mathrm{M}_{112 \mathrm{~m}}$ & \multirow{3}{*}{$\ldots$} & $\mathrm{M}_{1 \ln 1}$ & $\ldots$ & $\mathrm{M}_{11 \mathrm{~nm}}$ & $\mathrm{O}_{11}$ & \multirow{3}{*}{$\mathrm{O}_{\mathrm{T} 1}$} \\
\hline & & & \multirow{2}{*}{$\frac{\ldots}{M_{1 \mathrm{~m} 21}}$} & & \multirow{2}{*}{$\ldots$} & & \multicolumn{2}{|l|}{$\ldots$} & \multicolumn{2}{|l|}{$\ldots$} & \\
\hline & & & & $\ldots$ & & & $\mathrm{M}_{1 \mathrm{mn} 1}$ & $\ldots$ & $\mathrm{M}_{1 \mathrm{mnm}}$ & $\overline{\mathrm{O}_{1 \mathrm{~m}}}$ & \\
\hline $\mathrm{M}_{2111}$ & $\ldots$ & $\mathrm{M}_{211 \mathrm{~m}}$ & & & & & $\mathrm{M}_{2 \ln 1}$ & $\ldots$ & $\mathrm{M}_{21 \mathrm{~nm}}$ & $\mathrm{O}_{21}$ & \\
\hline$\ldots$ & & $\ldots$ & & $\ddots$ & & $\ldots$ & $\ldots$ & & $\ldots$ & $\ldots$ & $\mathrm{O}_{\mathrm{T} 2}$ \\
\hline $\mathrm{M}_{2 \mathrm{~m} 11}$ & $\ldots$ & $\mathrm{M}_{2 \mathrm{mlm}}$ & & & & & $\mathrm{M}_{2 \mathrm{mn} 1}$ & $\ldots$ & $\mathrm{M}_{2 \mathrm{mnm}}$ & $\mathrm{O}_{2 \mathrm{~m}}$ & \\
\hline & $\cdots$ & & & $\ldots$ & & 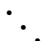 & & $\ldots$ & & & \\
\hline $\mathrm{M}_{\mathrm{n} 111}$ & $\ldots$ & $\mathrm{M}_{\mathrm{n} 1 \mathrm{~m}}$ & $\mathrm{M}_{\mathrm{n} 121}$ & $\ldots$ & $\mathrm{M}_{\mathrm{n} 12 \mathrm{~m}}$ & & & & & $\mathrm{O}_{\mathrm{n} 1}$ & \\
\hline$\ldots$ & & $\ldots$ & $\ldots$ & & $\ldots$ & $\ldots$ & & $\ddots$ & & $\ldots$ & $\mathrm{O}_{\mathrm{Tn}}$ \\
\hline $\mathrm{M}_{\mathrm{nm} 11}$ & $\ldots$ & $\mathrm{M}_{\mathrm{nmlm}}$ & $\mathrm{M}_{\mathrm{nm} 21}$ & $\ldots$ & $\mathrm{M}_{\mathrm{nm2m}}$ & & & & & $\mathrm{O}_{\mathrm{nm}}$ & \\
\hline $\mathrm{D}_{11}$ & & $\mathrm{D}_{1 \mathrm{~m}}$ & $\mathrm{D}_{21}$ & & $\mathrm{D}_{2 \mathrm{~m}}$ & $\ldots$ & $D_{n 1}$ & $\ldots$ & $D_{n m}$ & & \\
\hline & $\mathrm{D}_{\mathrm{T} 1}$ & & & $\mathrm{D}_{\mathrm{T} 2}$ & & & & $\mathrm{D}_{\mathrm{Tn}}$ & & & \\
\hline
\end{tabular}
analyses the movement of passengers between the stations considered passenger cars $M \in \mathbb{R}^{n_{s} \times n_{s}}$,

Figure 2: $\quad$ New $M$ OD matrix.

where, each element of the alighting and boarding vectors and trip matrix must satisfy:

$$
D_{T J}=\sum_{k=1}^{m} D_{J k}
$$

The alighting element $\mathrm{D}_{\mathrm{TJ}}$ is the summation of alighting flows of $m$ traveller's cars at station $J$

$$
O_{T I}=\sum_{k=1}^{m} O_{I k}
$$


The boarding element $\mathrm{O}_{\mathrm{TI}}$ is the summation of alighting flows of $m$ traveller's cars at station $I$. The relationship between the matrix elements in original and new OD matrix follows,

$$
T_{I J}=\sum_{i=1}^{m} \sum_{j=1}^{m} M_{I i J j}
$$

subject to

$$
\begin{aligned}
& D_{I j}=\sum_{i=1}^{n_{s}} M_{I i J i} \forall_{j}=1 \ldots n_{s} \\
& O_{J i}=\sum_{j=1}^{n_{s}} M_{I i J j} \forall_{i}=1 \ldots n_{s}
\end{aligned}
$$

\section{Estimation of the elements of O-D matrix}

One way to estimate the travel's matrix elements is by assuming that the system is governed by the principle of maximum entropy. However, this estimation could be improved by incorporating information, even if it is inaccurate. The interaction and behaviour dynamics of different groups of passengers could be included by applying artificial intelligence-based techniques and more specifically Fuzzy Logic.

To apply this model of distribution based on the following assumptions:

a) $\boldsymbol{T}_{I J}, \boldsymbol{O}_{T I}$ and $\boldsymbol{D}_{T J}$ have little uncertainty within a planning horizon. Each element of vector $\boldsymbol{O}_{J i}$ or $\boldsymbol{D}_{\boldsymbol{I} j}$ can be represented as a function of the boarding vector $\boldsymbol{O}_{T I}$ or alighting vector $\boldsymbol{D}_{T J}$ respectively and a power function with exponents $\boldsymbol{C}_{\boldsymbol{D I j}}$ or $\boldsymbol{C}_{\boldsymbol{O} \boldsymbol{J \boldsymbol { i }}}$. Equations (7) and (8) approximate human reasoning for boarding and alighting distributions in cars,

$$
\begin{gathered}
D_{I j}=\frac{2^{C_{D I j}}}{\sum_{k=1}^{m} 2^{C_{D I k}}} D_{T J} \\
O_{J i}=\frac{2^{C_{O J i}}}{\sum_{k=1}^{m} 2^{C_{O J k}}} O_{T I}
\end{gathered}
$$

b) Each element of $\boldsymbol{M}_{\boldsymbol{I} I J j}$ trip matrix can be represented as $\boldsymbol{T}_{I J}$ function and a power function with exponent $\boldsymbol{C}_{M I i j j}$.

$$
M_{I i J j}=\frac{2^{C_{M i l i j}}}{\sum_{r=1}^{m} \sum_{s=1}^{m} 2^{C_{M I r J s}}} T_{I J}
$$


c) $\boldsymbol{C}_{\boldsymbol{D I j}}, \boldsymbol{C}_{\boldsymbol{O} \boldsymbol{J} \boldsymbol{i}}$ and $\boldsymbol{C}_{\boldsymbol{M I I J j}}$ come from a fuzzy inference engine based on the experience of an expert or railway's planner.

It is important to highlight here, that $\boldsymbol{C}_{\boldsymbol{D I j}}$ and $\boldsymbol{C}_{\boldsymbol{O} \boldsymbol{J} \boldsymbol{i}}$ exponents are related to the passengers preference to any train's coach at specific station for boarding or alighting. If these exponents are zero, the estimation becomes the maximum entropy estimation, while if you have a positive or negative number corresponds to a train's coach with low or high demand respectively.

On the other hand, the exponent $\boldsymbol{C}_{\boldsymbol{M I} \text { IJj }}$ sets the relative level of importance of the flow of passengers between train's coach $i$ at I station to train's coach $j$ at $\mathbf{J}$ station. Here, the maximum entropy estimate also corresponds to a value of zero and a negative or positive number corresponds to a low flow or high demand respectively.

\section{Dwelling time estimation}

The correct estimation of dwelling time is critical for an appropriate railway time table planning. It has been shown that this dwelling time is function of rolling stock, through passengers and passengers' flow on station platform.

The through passengers in car $k$ at station $l$ are defined by,

$$
M_{T l k}=\sum_{j=l+1}^{n} \sum_{I=1}^{l-1} \sum_{i=1}^{m} M_{I i j k}
$$

Harris and Anderson [8] proposed and demonstrated a formulation for dwelling time estimation. Even though, such formulation demands a good statistics in order to obtain an appropriate model, it is possible to approximate the time for opening and closing doors in train's coach $k$ at station $l$ as follows,

$$
t_{o c l k}=t_{o c m}+\left(1.5\left[1+0.9 \frac{M_{T l k}}{V_{c}}\right] \frac{D_{l k}^{a}}{n_{d}}\right)+\left(1.3\left[1+0.8 \frac{M_{T l k}}{V_{c}}\right] \frac{O_{l k}^{b}}{n_{d}}\right)+0.027 \frac{D_{l k} O_{l k}}{n_{d}^{2}}
$$

and consequently, the dwelling time is defined as:

$$
t_{d l}=\max \left(t_{o c l 1}, t_{o c l 2}, \ldots \ldots . ., t_{o c l m}\right)
$$

where,

$\mathrm{a}=0.7$

$\mathrm{b}=0.7$

$\mathrm{t}_{\mathrm{ocm}}=15$ seconds

$\mathrm{Vc}=$ Capacity of the hall (assuming $0.14 \mathrm{~m}^{2}$ per person)

$\mathrm{t}_{\mathrm{d} l}=$ Time to stop at the station $l$

$\mathrm{n}_{\mathrm{d}}=$ number of doors

$\mathrm{D}_{\mathrm{lk}=}$ alighting passengers from train's coach $k$ and station $l$

$\mathrm{O}_{\mathrm{lk}=}$ boarding passengers at train's coach $k$ and station $l$. 


\section{Fuzzy inference engine}

The exponents $\boldsymbol{C}_{\boldsymbol{D I j}}, \boldsymbol{C}_{\boldsymbol{O} \boldsymbol{O J}}$ and $\boldsymbol{C}_{\boldsymbol{M I I J j}}$ can be designed using the help of fuzzy logic. In this particular case it seems reasonable to use triangular membership functions (Figure 3). These exponents can be estimated by considering:

1. Stairs, elevators and other facilities are closer to some cars on the platform during the boarding process. Therefore, these cars present a greater flow of passengers. On the other hand, groups of more experienced passengers usually wait at more distant places on the platform knowing the exact location of the doors of the arriving train during peak hours [9].

2. Many passengers know the location of stairs, elevators and other facilities on the platform of the destination station. As a result, the cars that are closer to these facilities have a greater flow of passengers.

3. The level of car occupancy depends on the purpose of travel (work, studies, leisure, shopping, tourism, etc), gender and age [12].

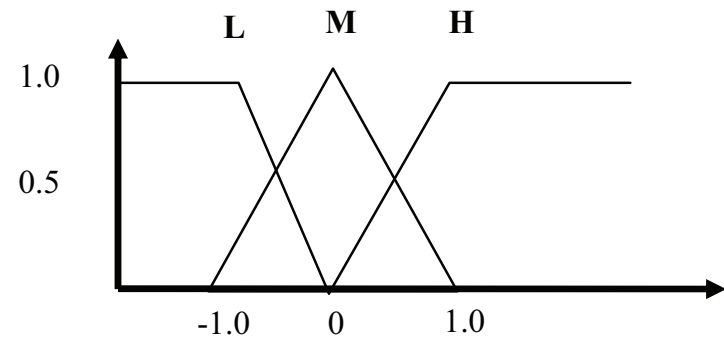

\begin{tabular}{|l|l|}
\hline $\mathbf{L}$ & Low level \\
\hline $\mathbf{M}$ & Medium level \\
\hline $\mathbf{H}$ & High level \\
\hline
\end{tabular}

Figure 3: $\quad$ Membership functions for $\boldsymbol{C}_{\boldsymbol{D I j}}, \boldsymbol{C}_{\boldsymbol{O} \boldsymbol{J I}}$ and $\boldsymbol{C}_{\boldsymbol{M I I J j} \text {. }}$

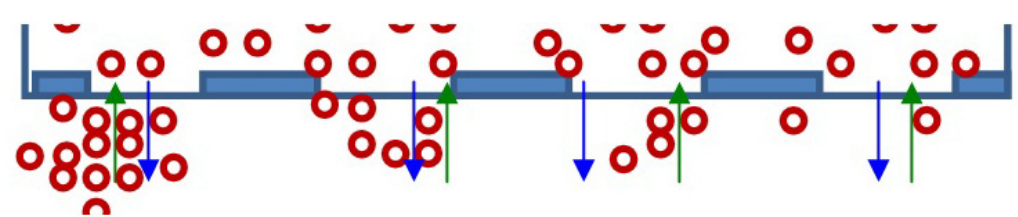

High

Medium

Low

level

level

level

Figure 4: Membership function levels for a train's coach.

The membership function levels of the alighting and boarding rates in each of doors car train are shown in figure 4. There are three levels of passenger's flow. Here, the high level approximates a great passenger's congestion; on the other hand the low level represents a weak passenger's flow. 


\section{Proposed algorithm}

a) Estimate the exponents $C_{D I j}, C_{O J i}$ and $C_{M I J j}$ basing on the inaccurate available information available using membership function levels for a train's coach.

b) Estimate attraction vectors and generation, $D_{I j}$ and $O_{J i}$, for the stations using the exponential equations (13) and (14)

$$
\begin{aligned}
& D_{I j}=\frac{2^{C_{D I j}}}{\sum_{k=1}^{m} 2^{C_{D I k}}} D_{T J} \\
& O_{J i}=\frac{2^{C_{O J i}}}{\sum_{k=1}^{m} 2^{C_{O J k}}} O_{T I}
\end{aligned}
$$

c) Estimate the trip matrix $\boldsymbol{M}_{I i J j}$, using,

$$
M_{I i J j}=\frac{2^{C_{M I i J j}}}{\sum_{r=1}^{m} \sum_{s=1}^{m} 2^{C_{M I r J s}}} T_{I J}
$$

d) Proceed to check if $O_{J i}=\sum_{j=1}^{n_{s}} M_{I i J j} \forall_{i}=1 \ldots n_{s}$. Consequently, if $\left|\sum_{j=1}^{n_{s}} M_{I i J j}-O_{J i}\right|<\varepsilon$ is true for a given level, it is accepted. Meanwhile, if not, the values of the matrix elements are corrected with

$$
M_{I i J j} \Leftarrow \frac{O_{i} M_{I i J j}}{\sum_{j=1}^{n_{s}} M_{I i J j}}
$$

e) Proceed to check if $D_{I j}=\sum_{i=1}^{n_{s}} M_{I i J i} \forall{ }_{j}=1 \ldots n_{s}$. Consequently, if $\left|\sum_{i=1}^{n_{s}} M_{I i J j}-D_{I j}\right|<\varepsilon$ is fulfilled, it is accepted. Meanwhile, if not, the values of the matrix elements are corrected with

$$
M_{I i J j} \Leftarrow \frac{D_{j} M_{I i J j}}{\sum_{i=1}^{n_{s}} M_{I i J j}}
$$


f) Proceed to check whether $T_{I J}=\sum_{i=1+m_{B}(I)}^{m_{E}(I)} \sum_{j=1+m_{B}(J)}^{m_{E}(J)} M_{I i j j}$, for all i, j, I, J. Consequently, if $\left|\sum_{i=1+m_{B}(I)}^{m_{E}(I)} \sum_{j=1+m_{B}(J)}^{m_{E}(J)} M_{i j m}-T_{I J}\right|<\varepsilon_{m}$ satisfied, it is accepted. Meanwhile, if not, the values of the matrix elements are corrected with

$$
M_{I I J j} \Leftarrow \frac{T_{I J} M_{I i J j}}{\sum_{i=1+m_{B}(I)}^{m_{E}(I)} \sum_{j=1+m_{B}(J)}^{m_{E}(J)} M_{I i J j}}
$$

g) Return to step d, if the tolerances are not satisfied.

h) Finally,

$$
\begin{gathered}
M_{T l k}=\sum_{j=l+1}^{n} \sum_{I=1}^{l-1} \sum_{i=1}^{m} M_{I i j k} \\
t_{o c l k}=t_{o c m}+\left(1.5\left[1+0.9 \frac{M_{T l k}}{V_{c}}\right] \frac{D_{l k}^{a}}{n_{d}}\right) W+\left(1.3\left[1+0.8 \frac{M_{T l k}}{V_{c}}\right] \frac{O_{l k}^{b}}{n_{d}}\right) W+0.027 \frac{D_{l k} O_{l k}}{n_{d}^{2}} \\
t_{d l}=\max \left(t_{o c l 1}, t_{o c l 2}, \ldots \ldots . ., t_{o c l m}\right)
\end{gathered}
$$

\section{Results for simulated data}

The simple following case illustrates how the proposed algorithm is applied. Let's consider a 3 station railway line with a three car train. This train has an O$\mathrm{D}$ matrix that not only considers the passenger flow between stations, but also between train cars in stations (please see figure 5).

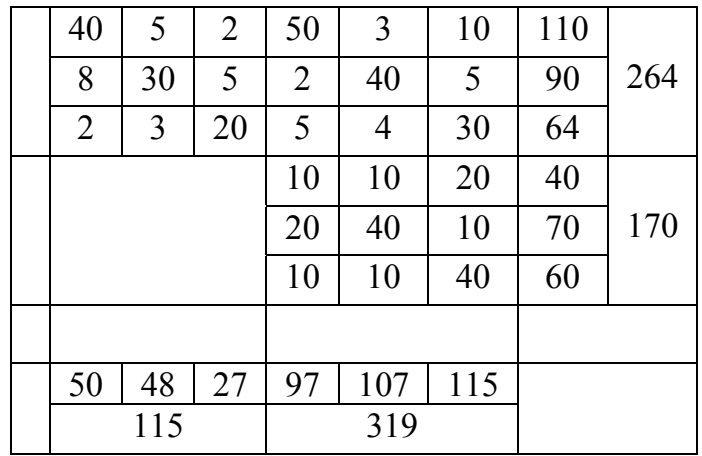

Figure 5: The real O-D trip matrix. 
Under these conditions the station dwelling time is 48 seconds. Commonly, the best information available about the elements of the last O-D matrix is imprecise. In this case, a general O-D matrix considering only passenger flow between stations and some general idea about the flow between cars considering passenger behavior (please see figure 6).

\begin{tabular}{|c|c|c|c|c|c|c|c|}
\hline $\mathrm{H}$ & $\mathrm{L}$ & $\mathrm{L}$ & $\mathrm{H}$ & $\mathrm{L}$ & $\mathrm{M}$ & $\mathrm{H}$ & \multirow{3}{*}{264} \\
\hline$\overline{\mathrm{L}}$ & $\mathrm{H}$ & $\mathrm{L}$ & $\bar{L}$ & $\mathrm{H}$ & $\mathrm{L}$ & $\mathrm{H}$ & \\
\hline \multirow[t]{4}{*}{$\mathrm{L}$} & $\mathrm{L}$ & $\mathrm{M}$ & $\mathrm{L}$ & $\mathrm{L}$ & $\mathrm{H}$ & $\mathrm{M}$ & \\
\hline & & & $\mathrm{M}$ & $\mathrm{M}$ & $\mathrm{M}$ & $\mathrm{M}$ & \multirow{3}{*}{170} \\
\hline & & & $\mathrm{M}$ & $\mathrm{H}$ & $\mathrm{M}$ & $\mathrm{H}$ & \\
\hline & & & $\mathrm{M}$ & $\mathrm{M}$ & $\mathrm{H}$ & $\mathrm{H}$ & \\
\hline & & & & & & & \\
\hline $\mathrm{H}$ & $\mathrm{H}$ & $\mathrm{M}$ & $\mathrm{H}$ & $\mathrm{H}$ & $\mathrm{H}$ & & \\
\hline & 115 & & & 319 & & & \\
\hline
\end{tabular}

\begin{tabular}{|l|l|l|}
\hline 115 & 149 & 264 \\
\hline & 170 & 170 \\
\hline & & \\
\hline 115 & 319 & \\
\hline
\end{tabular}

Figure 6: Imprecise O-D matrix.

As a result, the proposed algorithm estimates an approximated O-D matrix applying a Fuzzy Logic Inference motor (please see figure 7) and a station dwelling time of 51 seconds. Clearly, this station dwelling time obtained using the proposed algorithm has an acceptable error of $6.25 \%$ compared to the original trip matrix without the proposed algorithm.

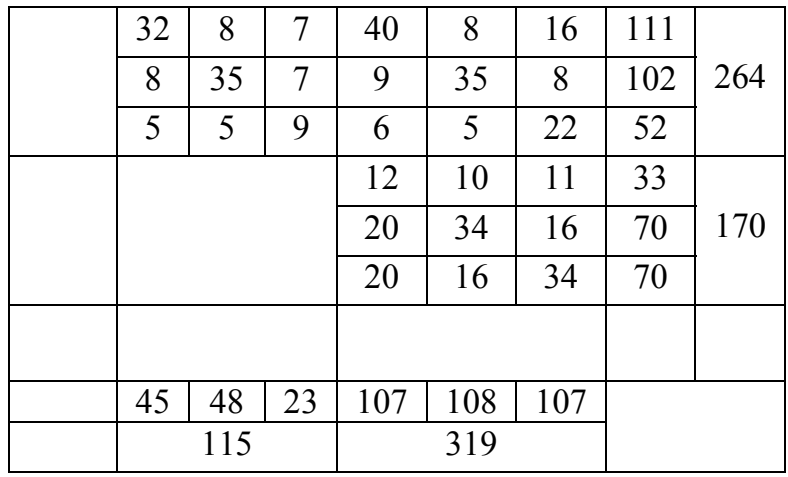

Figure 7: The resulting O-D matrix using the proposed algorithm.

\section{Conclusions}

Preliminary results have shown the potential and effectiveness of this new methodology. This new proposed algorithm has been developed using the help of fuzzy logic to predict the flow demand of passengers' trips, alighting and 
boarding time passenger cars in explicit stations. As a guideline for future research, it is interesting to improve the fuzzy inference engine, and proved it with real time data. Here, other sources of information could be incorporated without limiting the information just to one expert.

\section{Acknowledgements}

The authors of this paper want to express their gratitude to the National Secretary of Science and Technology (SENACYT) of the Government of the Republic of Panama for funding this study through the R\&D project "Methodologies and Performance Indicators of Railway Transport Systems" adjudged by a call for the promotion of R\&D activities (MDEPRB09-001) 2009-2011. Additionally, they want to thank the support received from the Technological University of Panama (UTP), the Intelligent Control Group (ICG) and the Research Centre in Railway Technologies (CITEF), both of the Polytechnic University of Madrid.

\section{References}

[1] Zhang Qi, Han Baoming, Li Dewei. Modeling and simulation of passenger alighting and boarding movement in Beijing metro stations. Transportation Research Part C 16 (2008) 635-649. 2008.

[2] Harris, N. Train Boarding and Alighting Rates at High Passenger Loads. Journal of Advanced Transportation, Vol. 40, No. 3, pp. 249-263. 2006.

[3] S. Kikuchi and D. Miljkovic. Method To Preprocess Observed Traffic Data for Consistency Application of Fuzzy Optimization Concept. Transportation Research Record 1679 Paper No. 99-0129 73. 1999.

[4] Aldian, A. and Taylor, M. Fuzzy multicriteria analysis for inter-city travel demand modelling. Journal of the Eastern Asia Society for Transportation Studies, Vol. 5, October, 2003.

[5] Watson, J. R. and Prevedouros, P. D. Derivation of Origin-Destination Distributions from Traffic Counts Implications for Freeway Simulation. Transportation Research Record: Journal of the Transportation Research Board, No. 1964, Transportation Research Board of the National Academies, Washington, D.C., 2006, pp. 260-269. 2006.

[6] Cheng, Y.H. and Yang, Li-An. A Fuzzy Petri Nets approach for railway traffic control in case of abnormality: Evidence from Taiwan railway system. Expert Systems with Applications 36 (2009) 8040-8048. 2009.

[7] A. Berbey, R. Caballero, R. Galán, J. D. Sanz Bobi. A fuzzy logic inference approach for the estimation of the passengers flow demand. Proceedings of the International Conference on Fuzzy Computation and 2nd International Conference on Neural Computation, pages 125-129. Valencia, Spain. October. 2010

[8] Harris, N., Anderson, R. An international comparison of urban rail boarding and alighting rates. Proc. IMechE Vol. 221 Part F: J. Rail and Rapid Transit. 2007. 
[9] Paul B.L. Wiggenraad. Alighting and boarding times of passengers at Dutch railway stations. Analysis of data collected at 7 railway stations in October 2000. TRAIL Research School. December 2001.

[10] Magnolo, E., Manenti, L., Manzoni, S., Sartori, F. Towards a MAS Model for Crowd Simulation at Pop-Rock Concerts Exploiting Ontologies and Fuzzy Logic. 2009.

[11] Ortúzar, J.D., Willumsen, L.G. Modelling transport. John Wiley \& Sons Inc 2011.

[12] Daamen, W.; Hoogendoorn, S. Research on pedestrian traffic flow in the Netherlands. In proceedings Walk 21 IV (pp. 101-117) Portland, Oregon. United States. Walk 21 Conference. 2003.

[13] C. Eksin, M. Güzelkaya, E. Ye_il, I. Eksin. Fuzzy Logic Approach to Mimic Decision Making Behavior of Humans in Stock Management Game. Proceedings of the 2008 System Dynamics Conference 2008.

[14] Railway Consultancy. Station capacity at Norreport. Report for Danish Government, 2000, available from http://www.trm.dk/sw677.asp(available online on the Danish Government, website). 2000. 